\title{
Diabetes and Periodontitis - A Socioeconomic Disease?
}

\author{
Pooja Palwankar ${ }^{1}$, Shourya Tandon², Vikram Blaggana ${ }^{3}$, Drishti Palwankar ${ }^{4}$, Akshat Sachdeva ${ }^{5}$ \\ ${ }^{1}$ Department of Periodontology, Manav Rachna Dental College, MRIIRS, Faridabad, Haryana, India, \\ ${ }^{2}$ Department of Public Health Dentistry, SGT Dental College, Research Institute \& Hospital, Gurgaon, \\ Haryana, India, ${ }^{3}$ Department of Periodontology, Inderprastha Dental College \& Hospital, Ghaziabad, \\ Uttar Pradesh, India, 4, 5 Department of Conservative Dentistry and Endodontics, Sudha Rastogi \\ College of Dental Sciences and Research, Faridabad, Haryana, India.
}

\section{ABSTRACT}

Periodontitis is a chronic inflammation of periodontal tissues. It is a slow progressing infectious disease. Periodontitis is recognized as the sixth complication of diabetes. Periodontitis and diabetes are chronic diseases. It has a bilateral relationship with diabetes mellitus. Socioeconomic status encompasses income, educational status of head of the family, and family occupation.

To upgrade and assess the knowledge concerning the association of periodontitis with diabetes and socioeconomic status, conclusions derived from the case - control studies, epidemiological studies and cross-sectional studies indexed in PubMed / Medline, Scopus journals during the period of 2010 - 2020 were analysed. This article elucidates 1) Whether there is a relationship between periodontitis and diabetes and vice-versa? 2) Association between periodontitis and socioeconomic status? 3) Relationship between socioeconomic status, diabetes, and periodontitis? After a thorough evaluation of the literature, it was found that there is a link between diabetes with periodontitis and vice-versa, socioeconomic status with periodontitis and diabetes, and thus there is a direct association of periodontitis with diabetes and socioeconomic status. Diabetes and socioeconomic status are the prime risk factors of periodontitis.

The education and motivation regarding periodontal health in all socioeconomic groups, pre-diabetes, and diabetics with periodontitis in the population would yield a better oral health quality of life. With the changes in socioeconomic status scale in each year, the need for reviewing the association between periodontitis, diabetes, and socioeconomic status in India becomes imperative.

\section{KEY WORDS}

SES - Socioeconomic Status, IDDM - Insulin - Dependent Diabetes Mellitus, NIDDM Non - Insulin - Dependent Diabetes Mellitus, IL - 6 - Interleukin - 6, IL - $1 \beta$ - Interleukin 1 Beta, TNF $\alpha$ - Tumour Necrosis Factor Alpha
Corresponding Author: Dr. Pooja Palwankar, Professor and Head, Department of Periodontology, Manav Rachna Dental College, MRIIRS, Faridabad - 121001, Haryana, India. E-mail:poojapalwankar@gmail.com

DOI: $10.14260 / j e m d s / 2021 / 474$

How to Cite This Article: Palwankar P, Tandon S, Blaggana V, et al. Diabetes and periodontitis - a socioeconomic disease? J Evolution Med Dent Sci 2021;10(30):2320-2324, DOI: $10.14260 / \mathrm{jemds} / 2021 / 474$

Submission 16-03-2021, Peer Review 24-05-2021, Acceptance 31-05-2021, Published 26-07-2021.

Copyright (c) 2021 Pooja Palwankar et al. This is an open access article distributed under Creative Commons Attribution License [Attribution 4.0 International (CC BY 4.0)] 


\section{BACKGROUND}

Periodontitis is the inflammation of the periodontium. It is known to affect human beings since ancient times, irrespective of race, ethnicity, etc. It is associated with loss of attachment, bone loss, mobility of one or more teeth. Many risk factors directly or indirectly contribute to cause chronic periodontitis,

1. Local factors in the form of dental plaque and calculus.

2. Systemic factors - Diabetes, Cardiovascular diseases, rheumatoid arthritis

3. Environmental and Behavioural factors - smoking, stress.

4. Genetic factors- The progression rate of periodontitis is deferred in healthy individuals in contrast to the individuals with systemic disease. The socioeconomic scale is an amalgamation of occupation, education of the head of the family, and family income, which gets updated with the changes in all India consumer price index (AICPI) every year.

The rationale of the study - Periodontitis being a chronic inflammatory disease can lead to the breakdown of collagen fibres through the release of inflammatory cytokines thus may lead to the loss of tooth. Periodontal examination of prediabetes and diabetic patients and different socioeconomic groups could lead to the prevention and arrest of periodontal disease in an incipient state, thus reducing the burden on global health systems.

This research intended to evaluate the relation of diabetes with periodontitis, socioeconomic status with diabetes, and periodontitis.

\section{METHODS}

The current literature on diabetes, socioeconomic status, and periodontitis was critically analysed using a digital database from the year 2010 to 2020. The cross-sectional studies, review articles, epidemiological studies in the PubMed / Medline and Scopus indexed journals were scrutinized, focussing on the dependent and independent variables, diabetes, and socioeconomic status. Keywords used in the search are "Periodontitis", "socioeconomic status" and "diabetes".

\section{DIABETES}

It is one of the systemic conditions which can accelerate the severity of periodontitis. Diabetes is an autoimmune disease that affects the individual with a sedimentary lifestyle. The majority of diabetic patients fall broadly into two categories type 1 and type 2 diabetics. The International Diabetes Federation (IDF) has reported the national, regional, and global occurrence of diabetes. Diabetes would take the epidemic status globally with the prediction. In 2009 it was estimated that 285 million people with diabetes (Type I Diabetes and Type II Diabetes) would increase to 366 million in 2011, 382 million in 2013, 415 million in 2015, and 425 million in 2017. The global diabetes prevalence in 2019 was estimated to be $9.3 \%$ (463 million people), rising to $10.2 \%$ (578 million) by 2030 and $10.9 \%$ (700 million) by 2045 . The prevalence is higher in urban $(10.8 \%)$ than rural $(7.2 \%)$ areas, and in high-income (10.4\%) than low-income countries $(4.0 \%)$.

One in two (50.1\%) people living with diabetes do not know that they have the disease. The global prevalence of impaired glucose tolerance was estimated to be $7.5 \%$ (374 million) in 2019 and projected to reach $8.0 \%$ (454 million) by 2030 and $8.6 \%$ (548 million) by 2045.1 According to the Diabetes Atlas Committee among the classification of diabetes, type 1 diabetes, or Insulin-dependent diabetes mellitus (IDDM), is prevalent in children, teenagers, and young adults which may lead to increased periodontal destruction especially in uncontrolled diabetes. Type - II diabetes or non insulin-dependent diabetes mellitus (NIDDM).2,3

Periodontitis is an inflammatory disease of the tissues that support the teeth, caused by specific microorganisms or groups of microorganisms, which results in progressive inflammatory destruction of the periodontal ligament and the alveolar bone and the formation of periodontal pockets, or receding gums, or both. The clinical sign that distinguishes gingivitis from periodontitis is the loss of attachment and inflammation with high prevalence. 4,5

Today, the diseases that affect the periodontium are classified according to the proposal made by Armitage in 1999. This classification divides the different clinical entities into the following major groups: gingival diseases, chronic periodontitis, aggressive periodontitis, periodontitis as a manifestation of systemic diseases, necrotizing periodontal diseases, abscesses of the periodontium, periodontitis associated with endodontic lesions, and developmental or acquired deformities and conditions. ${ }^{6,7}$ The clinical signs used to diagnose the periodontal disease are probing depth, bleeding on probing and clinical attachment level.

\section{DIABETES AND PERIODONTITIS}

Type II diabetes and chronic periodontitis are more likely to affect adults. ${ }^{8}$ The harmonious effect of local factors and host response in diabetics leads to severe periodontal destruction, which may not be possible to manage with scaling and root planing stand-alone therapy without controlling the glycaemic levels. Diabetes mellitus plays a major role in not only the initiation but also the progression of periodontitis.9,10 The salivary and gingival crevicular fluid have been shown to have inflammatory mediators and circulating cytokines in the form of interleukin-6 in diabetic patients with chronic periodontitis.11,12 The poorly controlled diabetes in the presence of local factors like dental plaque releases free radicals leading to less antioxidant activity, triggers the host cell neutrophils, lymphocytes and macrophages to release IL $1 \beta$, IL - 6, prostaglandins, and TNF - $\alpha$. The release of these cells stimulate Matrix Metalloproteinases, leading to the breakdown of collagen and bone loss proceeding to periodontitis. ${ }^{13,14}$ Kesavan $\mathrm{R}$ et al. concluded in their study that there is a significant association between diabetes and periodontitis, although the oral hygiene status in nondiabetics was not superior to diabetics. ${ }^{15}$ The study done in the rural population of Vellore found that individuals with type 2 diabetes had a higher prevalence of periodontitis. ${ }^{16}$ The literature study done on the database on RCT concluded that diabetes affects periodontitis and reverse periodontal disease 
has a detrimental effect on diabetes and its related complications. ${ }^{17}$ The poorly controlled diabetics have a significantly higher prevalence of periodontitis than controlled diabetics.

This could be attributed to excessive free radicals and less antioxidant activity. The pathogenesis related to periodontal tissues in diabetics is because of the altered host susceptibility due to the accumulation of advanced glycation end products (AGE). The poorly controlled diabetic patients are more prone to develop periodontitis and this in turn, interferes with the glycaemic control of diabetics. The treatment of periodontitis is beneficial in reducing glycaemic blood sugar levels. The incidence of microalbuminuria and end stage renal disease is increased twice or thrice in diabetic patients with severe periodontitis than diabetic patients without periodontitis. There is an increasing risk of cardio renal mortality in diabetic patients with severe periodontitis than diabetic patients without severe periodontitis. ${ }^{18}$ The uncontrolled HbA1c levels and elevated cardiovascular risk factors significantly increase the severity of periodontitis in type 2 diabetes mellitus. ${ }^{19}$ The aetiology associated with periodontal disease is multifactorial. The risk factors for periodontitis are plaque, calculus and gingivitis, although studies have shown that these clinical factors alone are insufficient to cause loss of attachment. Other factors related to periodontal disease are genetic factors, ethnicity, advanced age, male gender, depression, stress, smoking, traumatic occlusion, osteoporosis, the presence of red - complex bacteria (Porphyromonas gingivalis, Tanarella forsythus, Treponema denticola) and Prevotella intermedia and lack of dental care.20

\section{SOCIO-ECONOMIC STATUS}

The socioeconomic status (SES) is an economic and sociological combined total measure of a person's work experience and an individual's or family's economic and social position with others. It is determined based on various variables namely education, occupation and income level. The socioeconomic scale considers income as the basis for its calculation and it needs to be updated with the changes in the All India Consumer Price Index regularly. The modified Kuppuswamy scale is widely used to measure SES in the country with urban and rural areas. ${ }^{21,22}$ There are different scales to measure socioeconomic status namely B. G Prasad classification 1961, Udai Parikh scale 1964, Kulshrestha scale 1972, Kuppuswamy scale 1976, Shrivastava scale 1978, Bharadwaj scale 2001, S. C Tiwari et al. 2005, O. P Agarwal et al. 2005, Gaur scale 2013, and Satya Priya et al. 2015, Khairnar R et al. 2016 Tullika Singh 2017, Saleem SM 2018, Saleem SM 2019 , etc. There was a need for simultaneous updating of all socio-economic classifications which consider income as a parameter and the updated socio-economic scales were used by researchers in community health studies to determine the socioeconomic status of the study subjects precisely. ${ }^{23,24}$ The Kuppuswamy's socioeconomic status is an important tool in hospital and community based research in India which was originally proposed in 1976. There is a need for realizing the value of this important tool, and demand to update regularly, as the price index is increasing (as this scale is consumer price index-based).25,26 The per capita income of citizens decides the socioeconomic status scale of the population. The periodic changes in the consumer price index of goods and inflation warrant updating income-based socioeconomic status. ${ }^{27,28}$ There is an inverse relationship between socioeconomic status and mental health problems in children and adolescents. A decrease in socioeconomic status leads to the development of more mental health problems. ${ }^{29}$ People with low socioeconomic status have an increased prevalence of chronic diseases. The low socioeconomic status is associated with 18 out of 56 systemic conditions. ${ }^{30}$

\section{SOCIOECONOMIC STATUS AND PERIODONTITIS}

Socioeconomic status is one of the risk determinants for periodontal disease. ${ }^{31}$ Gingivitis and poor oral hygiene can be related to lower socioeconomic status. The prevalence is higher in urban $(10.8 \%)$ than rural $(7.2 \%)$ areas, and in high -income $(10.4 \%)$ than low-income countries $(4.0 \%)$. The literature data is conflicting, with some authors reporting better periodontal conditions with higher socioeconomic status while others have reported that there is no significant association between socioeconomic status and periodontitis. ${ }^{32}$ Individuals with low socioeconomic status and educational status are prone to periodontitis. ${ }^{33}$

However, when oral hygiene is taken care of, the association between socioeconomic status and periodontitis could not be proved. This study deferred from the other studies, where oral hygiene was proved to be poor in low socioeconomic status. ${ }^{11}$ Increased risk has been recorded in those population groups who suffered from poor nutrition in childhood, rural to urban migrants, educational status of the population. ${ }^{34}$ Bertoldi reported that there is a direct relationship between socioeconomic status and periodontitis. $^{35}$

\section{DIABETES AND SOCIOECONOMIC STATUS}

Socioeconomic status has a substantial effect on the blood sugar level of black men with diabetes. ${ }^{36}$ Individuals belonging to lower socio-economic status are more likely to develop diabetes, especially in poor employment status. ${ }^{37}$ The research in developing and developed countries have stated in their respective studies that low education, low income, and minority status are high-risk factors for diabetes mellitus. Socioeconomic status is one of the risk determinants along with genetic factors and other environmental factors like stress, smoking. ${ }^{38}$

There was a significant association between diabetes and socioeconomic status in women. ${ }^{39}$ The risk of developing diabetes was associated with low socioeconomic status in high, middle, and low-income countries.

The strength of the association was consistent in high income countries. ${ }^{40,41}$ Low education level and occupational position were associated with a threefold risk of prevalent diabetes in a cross-sectional cohort of middle-aged men and women. The lower socioeconomic group faces various challenges with diabetes, and they are at risk of contracting the disease, secondly, they are at high risk of having diabetes and are undiagnosed and remain untreated; thirdly they have difficulty in self-management of disease. ${ }^{42}$ 
On reviewing various cross-sectional studies, it was found that there was an association of socioeconomic status with diabetes in the lower socioeconomic groups in the Indian population as compared to higher income groups and similarly, there was a link between diabetes and periodontitis because of impaired host response in both the diseases.

\section{DISCUSSION}

Periodontal inflammation evokes the disturbance in the subgingival environment leading to disruption of homeostasis, creating a niche for the growth of anaerobic gram-negative bacteria leading to release of inflammatory cytokines, IL - $1 \beta$, IL - 6, Prostaglandins, MMPs leading to the destruction of alveolar bone and collagen destruction. ${ }^{43,44}$ Similar changes occur in diabetic patients with poor oral hygiene which is more prevalent in low socioeconomic status groups of the population. The analysis of the literature revealed that there is an association between periodontitis with metabolic diseases such as diabetes, and also a link between diabetes and socioeconomic status.

The occupation along with oral health quality of life also plays a vital role in the prevalence of periodontitis with adequate access to oral hygiene measures. ${ }^{45}$ The treatment of metabolic disease, namely diabetes should include periodontal disease prevention and treatment strategies in daily practice which would thereby decrease the prevalence and incidence of periodontal diseases. The prevalence of periodontitis is high in lower education and family income status. Socioeconomic status has been shown to influence the mental and systemic health. ${ }^{46}$ The medical, dental, and allied science clinicians should gain an insight into the link between periodontal and systemic diseases and their associated risk factors such as low socioeconomic status and other environmental factors. They should work in harmony with the periodontist or dental care specialist to treat the periodontal disease in its incipient stage.

\section{CONCLUSIONS}

Periodontal disease is the most prevalent infectious condition, but is treatable and preventable if taken care of in its primitive stages. Thus, it is concluded that low socioeconomic status and diabetes are true risk factors for periodontitis. The strategies to prevent and control the risk factors associated with periodontitis should be planned and executed effectively. The screening for diabetes in individuals with periodontal disease and examination of periodontal tissues in diabetics should be made mandatory in private clinics and health care delivery systems.

The emphasis should be given to periodontitis patients of lower socioeconomic status who should be educated and motivated about oral hygiene measures through various sources of mass media. A regular preventive program should be conducted for examination and treatment of patients belonging to various socioeconomic status groups. The signs and symptoms of periodontitis should be made aware to the general population, medical practitioners, and endocrinologists per se. There should be screening for pre- diabetes and periodontal diseases in the early stages in low socio-economic groups and less literate population which in turn can cut down the prevalence of diabetes and periodontitis and thus taking the edge off financial burden on the health system.

Financial or other competing interests: None.

Disclosure forms provided by the authors are available with the full text of this article at jemds.com.

\section{REFERENCES}

[1] Saeedi P, Petersohn I, Malanda B, et al. Global and regional diabetes prevalence estimates for 2019 and projections for 2030 and 2045: results from the International Diabetes Federation Diabetes Atlas, $9^{\text {th }}$ edition. Diabetes Res Clin Pract 2019;157:107843.

[2] Punthakee Z, Goldenberg R. Definition, classification and diagnosis of diabetes, prediabetes and metabolic syndrome. Can J Diabetes 2018;42(Suppl 1):S10-5.

[3] American Diabetes Association. Diagnosis and classification of diabetes mellitus. Diabetes Care 2009;32:S62-7.

[4] Armitage GC. Clinical evaluation of periodontal diseases. Periodontol 2000 1995;7:39-53.

[5] Almerich-Silla JM, Almiñana-Pastor PJ, Boronat-Catalá M, et al. Socioeconomic factors and severity of periodontal disease in adults (35-44 years). A cross sectional study. J Clin Exp Dent 2017;9(8):e988-94.

[6] Armitage GC. Development of a classification system for periodontal diseases and conditions. Ann Periodontol 1999;4(1):1-6.

[7] Highfield J. Diagnosis and classification of periodontal disease. Aust Dent J 2009;54(Suppl 1):S11-26.

[8] Sami W, Ansari T, Butt NS, et al. Effect of diet on type 2 diabetes mellitus: a review Int. J Health Sci (Qassim) 2017;11(2):65-71.

[9] Naiff P, Carneiro V, Guimarães MDC. Importance of mechanical periodontal therapy in patients with diabetes type 2 and periodontitis. Int J Dent 2018;2018:6924631.

[10] Heji ES, Bukhari AA, Bahammam MA, et al. Periodontal disease as a predictor of undiagnosed diabetes or prediabetes in dental patients. Eur J Dent 2021;15(2):216-21.

[11] Nazir MA. Prevalence of periodontal disease, its association with systemic diseases and prevention. Int J Health Sci (Qassim) 2017;11(2):72-80.

[12] Vincent RR, Appukuttan D, Victor DJ, et al. Oxidative stress in chronic periodontitis patients with type II diabetes mellitus. Eur J Dent 2018;12(2):225-31.

[13] Bajaj S, Khan A. Antioxidants and diabetes. Indian J Endocrinol Metab 2012;16(Suppl 2):S267-71.

[14] Patil VS, Patil VP, Gokhale N, et al. Chronic periodontitis in type 2 diabetes mellitus: oxidative stress as a common factor in periodontal tissue injury. J Clin Diagn Res 2016;10(4):BC12-6.

[15] Kesavan R, Chaly PE, Reddy VC, et al. Periodontal status among type II diabetic and nondiabetic individuals in Chennai, India: a comparative study. J Indian Assoc Public Health Dent 2015;13(4):393-8. 
[16] Nand KY, Oommen AM, Chacko RK, et al. Chronic periodontitis among diabetics and nondiabetics aged 3565 years, in a rural block in Vellore, Tamil Nadu: a crosssectional study. J Indian Soc Periodontol 2017;21(4):309. 14.

[17] Negrato CA, Tarzia O, Jovanovič L, et. Periodontal disease and diabetes mellitus. J Appl Oral Sci 2013;21(1):1-12.

[18] Preshaw PM, Alba AL, Herrera D, et al. Periodontitis and diabetes: a two-way relationship. Diabetologia 2012;55(1):21-31.

[19] Dhir S, Wangnoo S, Kumar V. Impact of glycemic levels in type 2 diabeties on periodontitis. Indian J Endocrinol Metab 2018;22(5):672-7.

[20] Socransky SS, Haffajee A, Cugini MA, et al. Microbial complexes in subgingival plaque. J Clin Periodontol 1998;25(2):134-44.

[21] Saleem SM. Modified kuppuswamy scale updated for year 2018. Paripex Indian J Res 2018;7(3):217-8.

[22] Saleem SM. Modified Kuppuswamy socioeconomic scale updated for the year 2019. Indian J Forensic Community Med 2019;6(1):1-3.

[23] Singh T, Sharma S, Nagesh S. Socio-economic status scales updated for 2017. Int J Res Med Sci 2017;5(7):3264-7.

[24] Dudeja P, Bahuguna P, Singh A, et al. Refining a socioeconomic status scale for use in community-based health research in India. J Postgrad Med 2015;61(2):77-83.

[25] Kumar BPR, Dudala SR, Rao AR. Kuppuswamy's socioeconomic status scale-a revision of economic parameter for 2012. Int J Res Dev Health 2013;1(1):2-4.

[26] Wani RT. Socioeconomic status scales-modified Kuppuswamy and Udai Pareekh's scale updated for 2019. J Family Med Prim Care 2019;8(6):1846-9.

[27] Reiss F. Socioeconomic inequalities and mental health problems in children and adolescents: a systematic review. Soc Sci Med 2013 A 2013;90:24-31.

[28] Mingo CA, Martin KR, Shreffler J, et al. Individual and community socioeconomic status: impact on mental health in individuals with arthritis. Arthritis 2014;2014:256498.

[29] Mair FS, Jani BD. Emerging trends and future research on the role of socioeconomic status in chronic illness and multimorbidity. Lancet Public Health 2020;5(3):e128-9.

[30] Balaji SK, Lavu V, Rao S. Chronic periodontitis prevalence and the inflammatory burden in a sample population from South India. Indian J Dent Res 2018;29(2):254-9.

[31] Borrell LN, Crawford ND. Socioeconomic position indicators and periodontitis: examining the evidence. Periodontol 2000 2012;58(1):69-83.

[32] Srivastava A, Mahmood SE, Srivastava PM, et al. Nutritional status of school-age children-a scenario of urban slums in India. Arch Public Health 2012;70(1):8.
[33] Bertoldi C, Lalla M, Pradelli JM, et al. Risk factors and socioeconomic condition effects on periodontal and dental health: a pilot study among adults over fifty years of age. Eur J Dent 2013;7(3):336-46.

[34] Assari S, Lankarani MM, Piette JD, et al. Socioeconomic status and glycemic control in type 2 diabetes: race by gender differences. Healthcare (Basel) 2017;5(4):83.

[35] Shrivastava A, Misra A, Gupta R, et al. Socioeconomic factors relating to diabetes and its management in India. J Diabetes 2016;8(1):12-23.

[36] Müller G, Hartwig S, Greiser KH, et al. Gender differences in the association of individual social class and neighbourhood unemployment rate with prevalent type 2 diabetes mellitus: a cross-sectional study from the DIABCORE consortium. BMJ Open 2013;3:e002601.

[37] Kinge KV, Supe AC. Association between socioeconomic status and diabetes mellitus in perimenopausal women in an urban slum of Mumbai. Int J Biomed Adv Res 2015:6(12):853-5.

[38] Carlsson S, Andersson T, Talbäck M, et al. Incidence and prevalence of type 2 diabetes by occupation: results from all Swedish employees. Diabetologia 2020;63(1):95-103.

[39] De Silva AP, De Silva SHP, Haniffa R, et al. Inequalities in the prevalence of diabetes mellitus and its risk factors in Sri Lanka: a lower middle income country. Int J Equity Health 2018;17(1):45.

[40] Agardh E, Allebeck P, Hallqvist J, et al. Type 2 diabetes incidence and socio-economic position: a systematic review and meta-analysis. Int $\mathrm{J}$ Epidemiol 2011;40(3):804-18.

[41] Abusleme L, Dupuy AK, Dutzan N, et al. The subgingival microbiome in health and periodontitis and its relationship with community biomass and inflammation. ISME J 2013;7(5):1016-25.

[42] Loos BG, Van Dyke TE. The role of inflammation and genetics in periodontal disease. Periodontol 2000 2020;83(1):26-39.

[43] Pinho RCM, De Melo Dias RSA, Bandeira F, et al. Impact of chronic periodontitis on the quality of life of individuals with and without diabetes. Health Sci J 2018;12(2):559.

[44] Suwannaphant K, Laohasiriwong W, Puttanapong N, et al. Association between socioeconomic status and diabetes mellitus: the national socioeconomics survey, 2010 and 2012. J Clin Diagn Res 2017;11(7):LC18-22.

[45] Persson GR. Diabetes and periodontal disease: an update for health care providers. Diabetes Spectrum 2011;24(4):195-8.

[46] Fenesy KE. Periodontal disease: an overview for physicians. Mt Sinai J Med 1998;65(5-6):362-9. 